\title{
The Comparison Between the MLE and Standard Bayes Estimators of the Reliability Function of Exponential Distribution
}

\author{
Mohammed Jamel Ali \\ Moham198999@gmail.com \\ Hazim Mansoor Gorgees \\ Hazim5656@yahoo.com \\ Department of Mathematics, Collage of Education for Pure Sciences Ibn AL-Haitham, University of \\ Baghdad, Baghdad, Iraq.
}

Article history: Received 10 June 2018, Accepted 1 October 2018, Publish January 2019

\begin{abstract}
In this paper, a Monte Carlo Simulation technique is used to compare the performance of MLE and the standard Bayes estimators of the reliability function of the one parameter exponential distribution. Two types of loss functions are adopted, namely, squared error loss function (SELF) and modified square error loss function (MSELF) with informative and noninformative prior. The criterion integrated mean square error (IMSE) is employed to assess the performance of such estimators.
\end{abstract}

Keywords: MLE, standard Bayes estimator, loss function, IMSE.

\section{Introduction}

The reliability theory is related with random happening of unwanted events or failure during the life of a physical or biological system [1]. Reliability is an essential feature of a system. Basic concepts associated with reliability has been known for a number of years, however, it has got greatest significance during the past decade as a consequence of the use of highly complex systems. In reliability theory, the exponential distribution plays an important role in life testing experiments. Historically, it was the first life time model for which statistical procedures were widely developed. Many researchers gave numerous results and generalized the exponential distribution as a life time distribution, particularly, in the field of industrial life testing. The exponential distribution is desirable because of its simplicity and its own features such as lacks memory and self-producing property. The probability density, cumulative distribution and reliability functions of one parameter exponential distribution are respectively defined as [2]:

The $f(t, \theta)=\theta e^{-\theta t}, \quad t, \theta>0$

Cumulative distribution function is given by:

$\mathrm{F}(\mathrm{t})=\operatorname{pr}(\mathrm{T} \leq \mathrm{t})=1-\mathrm{e}^{-\theta \mathrm{t}}$

$\mathrm{R}(\mathrm{t})=1-\mathrm{F}(\mathrm{t})=\mathrm{e}^{-\theta \mathrm{t}}$

\subsection{Maximum Likelihood Estimator}

Let $t_{1}, t_{2}, \ldots, t_{n}$ be the set of $\mathrm{n}$ random lifetimes from the one parameter exponential distribution then 
$\mathrm{L}\left(\theta ; \mathrm{t}_{1}, \mathrm{t}_{2}, \ldots, \mathrm{t}_{\mathrm{n}}\right)=\theta^{\mathrm{n}} \mathrm{e}^{-\theta \sum_{\mathrm{i}=1}^{\mathrm{n}} t_{i}}$

The value of $\theta$ maximize $\mathrm{L}$, also maximize $\ln \mathrm{L}$. Hence, by taking the natural logarithm for the likelihood function we get [3]

$\operatorname{lnL}\left(\theta ; \mathrm{t}_{1}, \mathrm{t}_{2}, \mathrm{t}_{\mathrm{n}}\right)=\operatorname{nln} \theta-\theta \sum_{\mathrm{i}=1}^{\mathrm{n}} t_{i}$

Differentiating the $\log$ likelihood function with respect to $\theta$ and setting the resultant derivative equal to zero, yield:

$\frac{\partial \ln L\left(; t_{1}, t_{2}, \ldots, t_{n}\right)}{\partial \theta}=\frac{n}{\Theta}-\sum_{i=1}^{n} t_{i}=0$

Therefore the MLE for $\Theta$ denoted by $\hat{\theta}_{M L E}$ is:

$\hat{\mathrm{\theta}}_{M L E}=\frac{n}{\sum_{i=1}^{n} t_{i}}=\frac{n}{T}$

Where we suppose that $=\sum_{i=1}^{n} t_{i}$.

Hence, the MLE of the reliability function will be

$\hat{R}(t)_{M L E}=e^{-\hat{\theta}_{M L E} t}$

\subsection{Standard Bayes Estimators}

The researchers employed two types of loss functions, namely, the squared error loss function (SELF) and modified squared error loss function (MSELF). The Bayes estimator of the parameter $\Theta$ is the value of $\Theta$ that minimize the risk function $R(\hat{\theta}, \theta)$ where [4]

$R(\hat{\theta}, \theta)=E[L(\hat{\theta}, \theta)]=\int_{\theta} L(\hat{\theta}, \theta) h(\theta \mid \underline{t}) d \theta$

In the case of squared error loss function, we have:

$L(\hat{\theta}, \theta)=(\hat{\theta}-\theta)^{2}$

Then, the risk function will be

$R(\hat{\theta}, \theta)=\int_{\theta}(\hat{\theta}-\theta)^{2} h(\theta \mid \underline{t}) d \theta$

$$
=\int_{\Theta} \hat{\theta}^{2} h(\Theta \mid \underline{t}) d \theta-2 \hat{\theta} \int_{\Theta} \theta h(\Theta \mid \underline{t}) d \theta+\int_{\Theta} \theta^{2} h(\Theta \mid \underline{t}) d \theta
$$

$R(\hat{\theta}, \theta)=\hat{\theta}^{2}-2 \hat{\theta} E(\theta \mid \underline{t})+E\left(\theta^{2} \mid \underline{t}\right)$

Differentiating $R(\hat{\theta}, \theta)$ with respect to $\hat{\theta}$ and setting the resultant derivative equal to zero, we get:

$2 \hat{\theta}-2 \mathrm{E}(\theta \mid \underline{t})=0$

Solving for $\hat{\theta}$ implies that

$\hat{\theta}_{S q}=\mathrm{E}(\boldsymbol{\theta} \mid \underline{\mathrm{t}})$

The Modified square error loss function is defined as [5]:

$\mathrm{L}(\hat{\theta}, \theta)=\theta^{\mathrm{r}}(\hat{\theta}-\theta)^{2}$

Where $r$ is a positive integer.

If (MSELF) is adopted, it can be in the same manner show that the Bayes estimator of $\theta$ is

$\hat{\theta}_{M S}=\frac{E\left(\theta^{r+1} \mid \underline{t}\right)}{E\left(\theta^{r} \mid \underline{t}\right)}$ 


\subsection{Posterior Density Based on Jeffrey's prior information}

Let us assume that $\Theta$ has non informative prior density. Jeffrey's (1961) developed a general rule for obtaining the prior distribution of $\theta$ [6]. He established that the single unknown parameter $\theta$ which is regarded as a random variable follows such a distribution that is proportional to the square root of the fisher information on $\theta$, that is [5]:

$g(\theta) \alpha \sqrt{I(\theta)}$

That is

$g(\theta)=c \sqrt{I(\theta)}$

Where $c$ is a constant of proportionality and $I(\theta)$ represent fisher information defined as follows:

$I(\theta)=-n E\left[\frac{\partial^{2} \ln f(t, \theta)}{\partial \theta^{2}}\right]$

If $g_{1}(\theta)$ denote Jeffrey's prior information then

$g_{1}(\theta)=c \sqrt{-n E\left(\frac{\partial^{2} \ln f(t ; \theta)}{\partial \Theta^{2}}\right)}$

For the exponential distribution we have

$\ln f(t, \theta)=\ln \theta-\theta t$

$\frac{\partial \operatorname{lnf}(\mathrm{t} ; \theta)}{\partial \theta}=\frac{1}{\theta}-\mathrm{t}$

The second derivative is

$\frac{\partial^{2} \operatorname{lnf}(\mathrm{t} ; \theta)}{\partial \Theta^{2}}=\frac{-1}{\Theta^{2}}$

Hence,

$E\left(\frac{\partial^{2} \ln f(t ; \theta)}{\partial \theta^{2}}\right)=\frac{-1}{\Theta^{2}}$

Substituting in equation (13) it follows that

$g_{1}(\Theta)=\frac{c}{\Theta} \sqrt{n}$

From Bayes theorem the posterior density function of $\Theta$ denoted by $h_{1}(\Theta \mid \underline{t})$ can be derived as [4]:

$h_{1}\left(\Theta \mid t_{1}, \ldots, t_{n}\right)=\frac{g_{1}(\Theta) L\left(\theta ; t_{1}, \ldots, t_{n}\right)}{\int_{0}^{\infty} g_{1}(\theta) L\left(\Theta ; t_{1}, \ldots, t_{n}\right) d \theta}$

$h_{1}\left(\theta \mid t_{1}, \ldots, t_{n}\right)=\frac{\Theta^{n-1} e^{-\Theta T}}{\int_{0}^{\infty} \theta^{n-1} e^{-\theta T} d \theta} \quad, \mathrm{T}=\sum_{i=1}^{n} t_{i}$

Hence, the posterior density function for $\theta$ based on Jeffery's prior information will be:

$h_{1}\left(\Theta \mid t_{1}, \ldots, t_{n}\right)=\frac{T^{n} \theta^{n-1} e^{-\Theta T}}{\Gamma(n)}$

The posterior density in equation (14) is defined identified as a density of the Gamma distribution, that is:

$\Theta \mathrm{I} t_{1}, t_{2}, \ldots, t_{n} \sim$ Gamma $\left(\mathrm{n}, \frac{1}{T}\right)$ with $\mathrm{E}(\Theta)=\frac{n}{T}$ and $\operatorname{var}(\Theta)=\frac{n}{T^{2}}$

$\Theta \sim \operatorname{Gamma}\left(\mathrm{n}, \frac{1}{\mathrm{~T}}\right)$ 


\subsection{Posterior Density Based on Gamma Prior Distribution}

Assuming that $\Theta$ has informative prior as Gamma distribution which takes the following form:

$g_{2}(\theta)=\frac{\beta^{\alpha} \Theta^{\alpha-1} e^{-\Theta \beta}}{\Gamma(\alpha)} \quad ; \theta>0, \beta>0, \alpha>0$

Where $\alpha, \beta$ are the shape parameter and scale parameter respectively.

The posterior density function is

$h_{2}(\Theta \mid \underline{t})=\frac{g_{2}(\Theta) L\left(\Theta ; t_{1}, \ldots, t_{n}\right)}{\int_{0}^{\infty} g_{2}(\Theta) L\left(\Theta ; t_{1}, \ldots, t_{n}\right) d \theta}$

Thus

$h_{2}(\theta \mid \underline{t})=\frac{P^{\alpha+n} \theta^{\alpha+n-1} e^{-\Theta P}}{\Gamma(\alpha+n)}$

Where $\mathrm{P}=(\beta+\mathrm{T})$

It can easily be noted that

$(\Theta \underline{t}) \sim \operatorname{Gamma}\left(\alpha+n, \frac{1}{P}\right)$ with $\mathrm{E}(\Theta)=\frac{\alpha+n}{P} \quad, \operatorname{Var}(\Theta)=\left(\frac{\alpha+n}{P^{2}}\right)$

\subsection{Bayes Estimator When (SELF) is Adopted}

a: The case of Jeffrey's prior information.

From equation (9) we found that:

$\hat{\theta}_{J S q}=\mathrm{E}(\theta \mid \underline{\mathrm{t}})=\int_{0}^{\infty} \theta h_{1}(\Theta \mid \underline{t}) d \theta$

$\hat{\theta}_{\mathrm{JSq}}=\frac{\mathrm{n}}{\mathrm{T}}$

Similarly, the Bayes estimator of the reliability function can be obtained:

as follows:

$\widehat{\mathrm{R}}(\mathrm{t})_{J S q}=\mathrm{E}(\mathrm{R}(\mathrm{t}) \underline{\mathrm{t}})=\int_{0}^{\infty} \mathrm{R}(\mathrm{t}) \mathrm{h}_{1}(\theta \mid \underline{\mathrm{t}}) \mathrm{d} \theta$

$\widehat{\mathrm{R}}(\mathrm{t})_{J S q}=\left(\frac{\mathrm{T}}{\mathrm{T}+\mathrm{t}}\right)^{\mathrm{n}}$

b: The case of Gamma prior distribution.

In this case we have

$\hat{\theta}_{\mathrm{GSq}}=\mathrm{E}(\theta \mathrm{I} \underline{\mathrm{t}})=\int_{0}^{\infty} \theta \mathrm{h}_{2}(\theta \mid \underline{\mathrm{t}}) \mathrm{d} \theta=\frac{\alpha+\mathrm{n}}{\mathrm{T}}$

The estimator of the reliability function can be obtained as:

$\hat{R}(t)_{G S q}=\mathrm{E}(\mathrm{R}(\mathrm{t}) \mid \underline{\mathrm{t}})=\int_{0}^{\infty} R(t) h_{2}(\theta \mid \underline{t}) d \theta=\left(\frac{P}{P+t}\right)^{\alpha+n}$

\subsection{Bayes Estimator When (MSELF) is Adopted}

a: The case of Jeffrey's prior information

From equation (11) we have:

$\hat{\theta}_{M S}=\frac{E\left(\theta^{r+1} \mid \underline{t}\right)}{E\left(\theta^{r} \mid \underline{t}\right)}$

The $r^{\text {th }}$ moment of $\theta$ I $\underline{t}$ can be evaluated as follows: 
$\mathrm{E}\left(\theta^{r} \mid \underline{t}\right)=\int_{0}^{\infty} \theta^{r} h_{1}(\theta \mid \underline{t}) d \theta$

Hence,

$\mathrm{E}\left(\Theta^{r} \mid \underline{t}\right)=\frac{\Gamma(n+r)}{\Gamma(n) T^{r}}$

Let us assume that $\mathrm{r}=1,3$ and $\hat{\mathrm{\theta}}_{\mathrm{JMS} 1}, \hat{\mathrm{\theta}}_{\mathrm{JMS} 2}$ represent the estimator of the parameter $\mathrm{\theta}$ corresponding to $\mathrm{r}=1,3$ respectively then by applying the formulas in (11) and (21) we get

$\hat{\theta}_{J M S 1}=\frac{E\left(\theta^{2} \mid \underline{t}\right)}{E(\theta \mid \underline{t})}=\frac{\frac{\Gamma(n+2)}{\Gamma(n) T^{2}}}{\frac{\Gamma(n+1)}{\Gamma(n) T}}=\frac{n+1}{T}$
$\hat{\theta}_{J M S 2}=\frac{E\left(\theta^{4} \mid \underline{t}\right)}{E\left(\theta^{3} \mid \underline{t}\right)}=\frac{\frac{\Gamma(n+4)}{\Gamma(n) T^{4}}}{\frac{\Gamma(n+3)}{\Gamma(n) T^{3}}}=\frac{n+3}{T}$

Similarly, the Bayes estimators of the reliability function can be obtained as follows

$\hat{R}(t)_{J M S 1}=\frac{E\left[\left((R(t))^{2} \mid \underline{t}\right)\right.}{E(R(t) \mid \underline{t})}$

$\hat{R}(t)_{J M S 2}=\frac{E\left[\left((R(t))^{4} \mid \underline{t}\right)\right]}{E\left[\left((R(t))^{3} \mid \underline{t}\right)\right]}$

Now, we have to determine each of $\mathrm{E}\left[\left((R(t))^{2} \mid \underline{t}\right)\right], \mathrm{E}\left[\left((R(t))^{3} \mid \underline{t}\right)\right]$ and $\mathrm{E}\left[\left((R(t))^{4} \mid \underline{t}\right)\right]$

$$
\begin{aligned}
E(R(t) \mid \underline{t}) & =\left(\frac{T}{T+t}\right)^{n} \\
\mathrm{E}\left((R(t))^{2} \mid \underline{t}\right) & =\int_{0}^{\infty}(R(t))^{2} h_{1}(\Theta \mid \underline{t}) d \theta \\
= & \left(\frac{T}{T+2 t}\right)^{n}
\end{aligned}
$$

By the same way we find that,

$$
\begin{aligned}
& \left.\mathrm{E}(R(t))^{3} \mid \underline{t}\right)=\left(\frac{T}{T+3 t}\right)^{n} \\
& \mathrm{E}(R(t))^{4}=\left(\frac{T}{T+4 t}\right)^{n}
\end{aligned}
$$

Hence,

$$
\begin{aligned}
\hat{R}(t)_{J M S 1}= & \frac{\left(\frac{T}{T+2 t}\right)^{n}}{\left(\frac{T}{T+t}\right)^{n}}=\left(\frac{T+t}{T+2 t}\right)^{n} \\
\hat{R}(t)_{J M S 2}= & \frac{\left(\frac{T}{T+4 t}\right)^{n}}{\left(\frac{T}{T+3 t}\right)^{n}}=\left(\frac{T+3 t}{T+4 t}\right)^{n}
\end{aligned}
$$

b: The case of Gamma prior distribution

From equation (11) we have:

$\hat{\theta}_{M S}=\frac{E\left(\Theta^{r+1} \mid \underline{t}\right)}{E\left(\Theta^{r} \mid \underline{t}\right)}$

The $r^{\text {th }}$ moment of elt $\underline{t}$ can be evaluated as follows:

$$
\begin{aligned}
& E\left(\Theta^{r} \mid \underline{t}\right)=\int_{0}^{\infty} \theta^{r} h_{2}(\Theta \mid \underline{t}) d \theta \\
& E\left(\theta^{r} \mid \underline{t}\right)=\frac{\Gamma(\alpha+n+r)}{\Gamma(\alpha+n) P^{r}}
\end{aligned}
$$

Let us assume that $r=1,3$ and $\hat{\theta}_{\mathrm{GMS} 1}, \hat{\theta}_{\mathrm{GMS} 2}$ represent the estimator of the parameter $\theta$ corresponding to $r=1,3$ respectively then by applying the formulas in (11) and (26) we get: 


$$
\begin{aligned}
\hat{\mathrm{\theta}}_{G M S 1} & =\frac{E\left(\theta^{2} \mid \underline{t}\right)}{\mathrm{E}\left(\theta^{\mathrm{\theta}} \underline{\mathrm{t}}\right)}=\frac{\frac{(\alpha+n)(\alpha+n+1)}{P^{2}}}{\frac{(\alpha+n)}{P}}=\frac{(\alpha+n+1)}{P} \\
\hat{\theta}_{G M S 2} & =\frac{E\left(\theta^{4} \mid \underline{t}\right)}{E\left(\theta^{3} \mid \underline{t}\right)}=\frac{\frac{(\alpha+n)(\alpha+n+1)(\alpha+n+2)(\alpha+n+3)}{P^{4}}}{\frac{(\alpha+n)(\alpha+n+1)(\alpha+n+2)}{P^{3}}} \\
= & \frac{\alpha+n+3}{P}
\end{aligned}
$$

Similarly, the Bayes estimators of the reliability function can be obtained as follows

$\hat{R}(t)_{G M S 1}=\frac{E\left[\left((R(t))^{2} \mid \underline{t}\right)\right]}{E(R(t) \mid t)}$

$\hat{R}(t)_{G M S 2}=\frac{E\left[\left((R(t))^{4} \mid \underline{t}\right)\right]}{E\left[\left((R(t))^{3} \mid \underline{t}\right)\right]}$

Now, we have to determine each of $\mathrm{E}\left[\left((R(t))^{2} \mid \underline{t}\right)\right], \mathrm{E}\left[\left((R(t))^{3} \mid \underline{t}\right)\right]$ and $\mathrm{E}\left[\left((R(t))^{4} \mid \underline{t}\right)\right]$

$$
\begin{aligned}
\mathrm{E}(\mathrm{R}(\mathrm{t}) \mid \underline{t}) & =\int_{0}^{\infty} R(t) h_{2}(\Theta \mid \underline{t}) d \Theta \\
= & \left(\frac{P}{P+t}\right)^{\alpha+n}
\end{aligned}
$$

$$
\begin{aligned}
\mathrm{E}\left((R(t))^{2} \mid \underline{t}\right) & =\int_{0}^{\infty}(R(t))^{2} h_{2}(\Theta \mid \underline{t}) d \Theta \\
& =\left(\frac{P}{P+2 t}\right)^{\alpha+n}
\end{aligned}
$$

Similarly, we find that

$$
\begin{aligned}
& \mathrm{E}\left((R(t))^{3} \mid \underline{t}\right)=\left(\frac{P}{P+3 t}\right)^{\alpha+n} \\
& \mathrm{E}\left((R(t))^{4} \mid \underline{t}\right)=\left(\frac{P}{P+4 t}\right)^{\alpha+n}
\end{aligned}
$$

Hence,

$$
\begin{aligned}
& \hat{R}(t)_{G M S 1}=\frac{E\left[\left((R(t))^{2} \mid \underline{t}\right)\right]}{E[(R(t) \mid \underline{t})]}=\frac{\left(\frac{P}{P+2 t}\right)^{\alpha+n}}{\left(\frac{P}{P+t}\right)^{\alpha+n}}=\left(\frac{P+t}{P+2 t}\right)^{\alpha+n} \\
& \hat{R}(t)_{G M s 2}=\frac{E\left[\left((R(t))^{4} \mid t\right)\right]}{E\left[\left((R(t))^{3} \mid \underline{t}\right)\right]}=\frac{\left(\frac{P}{P+4 t}\right)^{\alpha+n}}{\left(\frac{P}{P+3 t}\right)^{\alpha+n}}=\left(\frac{P+3 t}{P+4 t}\right)^{\alpha+n}
\end{aligned}
$$

\section{Simulation Study}

The simulation study was conducted in order to compare the performance of the maximum likelihood estimator (MLE) and Bayesian estimators of the reliability function $\mathrm{R}(\mathrm{t})$ of one parameter exponential distribution.

We adopted the integrated mean squared error (IMSE) as a criterion of comparison where

$$
\begin{aligned}
\operatorname{IMSE}[\hat{R}(t i)] & =\frac{1}{L} \sum_{i=1}^{L}\left[\frac{1}{n_{t}} \sum_{r=1}^{n_{t}}\left(\hat{R}_{i}\left(t_{r}\right)-R\left(t_{r}\right)\right)^{2}\right] \\
& =\frac{1}{n_{t}} \sum_{r=1}^{n_{t}} \operatorname{MSE}\left(\hat{R}\left(t_{r}\right)\right)
\end{aligned}
$$


Where $n_{t}$ is the random limits of $t_{r}$, using $\mathrm{t}=(0.1,0.2,0.3,0.4,0.5,0.6,0.7,0.8,0.9,1)$.

$\mathrm{L}$ is the number of replications which we assumed that $\mathrm{L}=1000$ in our study, $\hat{R}\left(t_{r}\right)$ is the estimator of $\mathrm{R}(\mathrm{t})$ at the $L^{\text {th }}$ replication.

The Bayesian estimators of $\mathrm{R}(\mathrm{t})$ are derived with respect to two loss function which are the Square error loss function (SELF) and Modified squared error loss function (MSELF), moreover, the informative and non-informative prior were postulated. The sample sizes $\mathrm{n}=10,50,100$ and 200 were chosen to represent small, moderate, large and very large sample sizes from the one parameter exponential distribution. The postulated values of the unique parameter $\Theta$ were $\Theta=0.5,1.5$ and the values of the parameters for Gamma prior were $\alpha=0.3,1$ and $\beta=1.2,3$.

The values assumed for the loss parameter of modified loss function were $r=1,3$. The results are presented in Tables (1-4).

Table 1. (IMSE) values of the reliability function estimators by using Jeffrey's prior information at $\Theta=0.5$

\begin{tabular}{|l|l|l|l|l|}
\hline \multicolumn{1}{|c|}{$\mathbf{1 0}$} & \multicolumn{1}{c|}{$\mathbf{5 0}$} & $\mathbf{1 0 0}$ & \multicolumn{1}{c|}{$\mathbf{2 0 0}$} \\
\hline Estimator & & & & \\
\hline MLE & 0.000574 & 0.004713 & 0.004083 & 0.002636 \\
\hline JSqu & 0.000526 & 0.004635 & 0.004060 & 0.002632 \\
\hline JMSq1 r=1 & 0.000450 & 0.004478 & 0.004014 & 0.002624 \\
\hline
\end{tabular}

Table 2. (IMSE) values of the reliability function estimators by using Jeffrey's prior information at $\Theta=1.5$

\begin{tabular}{|c|c|c|c|c|}
\hline Estimator & 10 & 50 & 100 & 200 \\
\hline MLE & 0.001008 & 0.003444 & 0.002316 & 0.001262 \\
\hline JSqu & 0.000911 & 0.003396 & 0.002309 & 0.001261 \\
\hline $\begin{array}{ll}\text { JMSq1 } & \mathrm{r}=1\end{array}$ & 0.000865 & 0.003293 & 0.002293 & 0.001260 \\
\hline $\begin{array}{ll}\mathrm{JMSq} 2 & \mathrm{r}=3\end{array}$ & 0.001262 & 0.003072 & 0.002258 & 0.001257 \\
\hline
\end{tabular}

Table 3. (IMSE) of the reliability function estimators by using Gamma prior information at $\Theta=0.5$

\begin{tabular}{|c|c|c|c|c|c|c|}
\hline \multicolumn{3}{|c|}{ Estimator $n$} & 10 & 50 & 100 & 200 \\
\hline \multirow{4}{*}{ GSqu } & \multirow[t]{2}{*}{$=0.3 \alpha$} & $\beta=1.2$ & 0.000404 & 0.004277 & 0.003938 & 0.002619 \\
\hline & & $\beta=3$ & 0.000335 & 0.003899 & 0.003748 & 0.002653 \\
\hline & \multirow[t]{2}{*}{$\alpha=1$} & $\beta=1.2$ & 0.000508 & 0.004395 & 0.003928 & 0.002605 \\
\hline & & $\beta=3$ & 0.000353 & 0.003945 & 0.003764 & 0.002563 \\
\hline \multirow{4}{*}{$\underset{r=1}{\text { GMSq1 }}$} & \multirow[t]{2}{*}{$\alpha=0.3$} & $\beta=1.2$ & 0.000359 & 0.004130 & 0.003890 & 0.002611 \\
\hline & & $\beta=3$ & 0.000324 & 0.003761 & 0.003700 & 0.002544 \\
\hline & \multirow[t]{2}{*}{$\alpha=1$} & $\beta=1.2$ & 0.000427 & 0.004247 & 0.003891 & 0.002597 \\
\hline & & $\beta=3$ & 0.000322 & 0.003808 & 0.003716 & 0.002555 \\
\hline \multirow{4}{*}{$\begin{array}{l}\text { GMSq2 } \\
\mathrm{r}=3\end{array}$} & \multirow[t]{2}{*}{$\alpha=0.3$} & $\beta=1.2$ & 0.000322 & 0.003846 & 0.003793 & 0.002595 \\
\hline & & $\beta=3$ & 0.000339 & 0.003495 & 0.003602 & 0.002526 \\
\hline & \multirow[t]{2}{*}{$\alpha=1$} & $\beta=1.2$ & 0.000329 & 0.003959 & 0.003785 & 0.002580 \\
\hline & & $\beta=3$ & 0.000300 & 0.003544 & 0.003619 & 0.002537 \\
\hline
\end{tabular}


Table 4. (IMSE) of the reliability function estimators by using Gamma prior information at $\Theta=1.5$

\begin{tabular}{|c|c|c|c|c|c|c|}
\hline \multicolumn{3}{|c|}{ Estimator $n$} & 10 & 50 & 100 & 200 \\
\hline \multirow[t]{4}{*}{ GSqu } & \multirow[t]{2}{*}{$=0.3 \alpha$} & $\beta=1.2$ & 0.000856 & 0.003020 & 0.002212 & 0.001250 \\
\hline & & $\beta=3$ & 0.001549 & 0.002459 & 0.002057 & 0.001225 \\
\hline & \multirow[t]{2}{*}{$\alpha=1$} & $\beta=1.2$ & 0.000713 & 0.003035 & 0.002214 & 0.001250 \\
\hline & & $\beta=3$ & 0.001096 & 0.002501 & 0.002045 & 0.001227 \\
\hline \multirow{4}{*}{$\underset{\mathrm{r}=1}{\mathrm{GMSq} 1}$} & \multirow[t]{2}{*}{$\alpha=0.3$} & $\beta=1.2$ & 0.001071 & 0.002913 & 0.002193 & 0.001248 \\
\hline & & $\beta=3$ & 0.001978 & 0.002352 & 0.002035 & 0.001223 \\
\hline & \multirow[t]{2}{*}{$\alpha=1$} & $\beta=1.2$ & 0.000816 & 0.002930 & 0.002195 & 0.001249 \\
\hline & & $\beta=3$ & 0.001451 & 0.002394 & 0.002023 & 0.001225 \\
\hline \multirow{4}{*}{$\begin{aligned} \mathrm{GMSq} 2 \\
\mathrm{r}=3\end{aligned}$} & \multirow[t]{2}{*}{$\alpha=0.3$} & $\beta=1.2$ & 0.001798 & 0.002688 & 0.002152 & 0.001244 \\
\hline & & $\beta=3$ & 0.002966 & 0.002135 & 0.001988 & 0.001217 \\
\hline & \multirow[t]{2}{*}{$\alpha=1$} & $\beta=1.2$ & 0.001353 & 0.002709 & 0.002155 & 0.001245 \\
\hline & & $\beta=3$ & 0.002319 & 0.002179 & 0.001975 & 0.001219 \\
\hline
\end{tabular}

\section{Simulation Results and Conclusions}

From our simulation study, the following results are clear

- From table 1: when $\Theta=0.5$ the Bayes estimator under modified squared error loss function when $r=3$ with Jeffrey's prior is the best comparing to other estimators for all sample sizes.

- From table 2: when $\theta=1.5$ the Bayes estimator under modified squared error loss function when $r=3$ with Jeffrey's prior is the best comparing to other estimators for sample sizes $(50,100,200)$ and in sample size $(10)$ the best is modified squared error loss function when $\mathrm{r}=1$.

- From table 3: when $\theta=0.5$ for $(n=10)$ the best is Bayes estimator under modified squared error loss function when $\mathrm{r}=3$ with Gamma prior $(\alpha=1, \beta=3)$ and for $(n=50,100,200)$ the Bayes estimator under modified squared error loss function when $\mathrm{r}=3$ with Gamma prior $(\alpha=0.3, \beta=3)$ is the best.

- From table 4: when $\Theta=1.5$ for $(n=10)$ the Bayes estimator under square error loss function with Gamma prior $(\alpha=1, \beta=1.2)$ is the best, and for $(n=50,200)$ the Bayes estimator under modified squared error loss function when $\mathrm{r}=3$ with Gamma prior $(\alpha=0.3, \beta=3)$ is the best and for $(n=100)$ the Bayes estimator under modified squared error loss function when $\mathrm{r}=3$ with Gamma prior $(\alpha=1, \beta=3)$ is the best.

- According to the simulation results we conclude that the Bayes estimator under modified squared error loss function with $r=3$ is superior to the performance of other estimators in almost cases that are studied in this paper, where the integrated mean squared error (IMSE) is employed as a criterion to assess the performance of such estimators. 


\section{References}

1. Meeker, W.Q.; Escobar, L.U.Statistical methods for Reliability Data, John Wiley \& sons. Inc. Canada. 1998.

2. Pugh, E.L. The best estimation of reliability in the exponential case, Journal of operation research. 1993,11.

3. AL-Timeemy, O.I. Estimation of Reliability function for inverse Gaussian Distribution model with Application by using Monte Carlo simulation, A thesis submitted to the college of science AL- Nahrain University for the Degree of Master of science in Applied Mathematics. 2008.

4. Dey, S. Bayesian Estimation of the parameter and Reliability Function of Inverse Rayleigh Distribution, Malaysian Journal of Mathematical science. 2012, 6, 1, 113 124.

5. Rasheed, H.A.; AL-Gazi, N.A.A. Bayesian Estimation for the Reliability Function of Pareto type I Distribution under Generalized square error loss function International Journal of Engineering and Innovative Technology (IJEIT). 2014, 4, 6, 33-41.

6. Kuo, W.; Zuo, M.J. Optimal Reliability Modeling principles and Applications. John Wiley \& sons Inc. Hoboken. New Jersey. 2003. 